\title{
EDITORIAL
}

\section{How long will chronic myeloid leukemia patients treated with imatinib mesylate live?}

\author{
J Hasford ${ }^{1}$, M Pfirmann ${ }^{1}$ and A Hochhaus ${ }^{2}$ \\ ${ }^{1}$ Leukemia and Lymphoma Biostatistics Group, IBE - Department of Medical Informatics, Biometry and Epidemiology, University \\ of Munich, Munich, Germany; and ${ }^{2}$ III. Medizinische Klinik, Fakultät für Klinische Medizin Mannheim, Universität Heidelberg, \\ Mannheim, Germany
}

Leukemia (2005) 19, 497-499. doi:10.1038/sj.leu.2403686

\section{How long will chronic myeloid leukemia (CML) patients treated with imatinib mesylate live?}

The stepwise approval of imatinib mesylate for the treatment of Philadelphia (Ph) chromosome-positive CML since 2001 occured mainly due to the remarkably high proportion of patients who achieved a partial or complete cytogenetic response. Whereas patients treated with interferon alfa (IFN) showed a major cytogenetic remission (MCR) rate between 7 and $37 \%,{ }^{1}$ the randomized IRIS trial ${ }^{2}$ demonstrated a remission rate of $85 \%$ for patients treated with imatinib within 18 months. As MCR is a surrogate measure only, patients, physicians and sickness funds alike want to know how long patients treated with imatinib will live compared to those treated with IFN, the pharmacotherapy with longest and best follow-up data.

There is clear evidence that MCR is a valid surrogate measure for the survival of low- and intermediate-risk patients treated with IFN. ${ }^{3,4}$ Assuming this for imatinib-treated patients too, it is possible to estimate their expected survival probabilities by combining the survival increment as observed in IFN-treated patients with MCR and the substantially higher rate of MCR achieved with imatinib.

From the Collaborative Prognostic Factors Project, ${ }^{5}$ our database comprised 658 IFN-treated patients with complete data on cytogenetic response and an observation of survival $>21$ months. A minimum of 20 metaphases had to be evaluated. Details on patient characteristics and the New CML score have been reported elsewhere. ${ }^{5}$ With regard to imatinib information on MCR as published by the IRIS trial was used. ${ }^{2}$

The percentage of MCR recorded up to 21 months was $26 \%$ with IFN. Survival probabilities in patients with and without MCR are shown in Table 1. The survival probabilities observed for a specific combination from New CML score (low, intermediate, high) and MCR (yes, no) in IFN-treated patients were assumed to be the same for patients treated with imatinib. The proportion of MCR among imatinib-treated patients was $85 \%$ at 18 months. $^{2}$ The same percentage was assumed at 21 months.

According to the $85 \%$ MCR rate, for estimating the survival probabilities of imatinib-treated patients, three samples were

Correspondence: Professor Dr J Hasford, Leukemia and Lymphoma Biostatistics Group, IBE - Department of Medical Informatics, Biometry and Epidemiology, Marchioninistr. 15, München D-81377, Germany; Fax: + 49897095 7482;

E-mail: has@ibe.med.uni-muenchen.de

Received 16 December 2004; accepted 27 December 2004 constituted by randomly drawing $85 \%$ of their patients of the IFN-treated patients with MCR and $15 \%$ out of the IFN-treated patients lacking MCR. The overall risk group distribution of the first simulated patient sample was chosen to be the same as for the 658 IFN patients: $41 \%$ low risk, $48 \%$ intermediate risk and $11 \%$ high risk. Regarding the second and third sample, simulations were performed only within the low-risk and intermediate-risk group, respectively.

For the total patient sample consisting of low-, intermediateand high-risk patients, median survival time with imatinib was not yet reached, whereas 75 months were observed for IFN (Figure 1). The estimated 10-year survival probability for imatinib was $51 \%$ compared to $26 \%$ with IFN.

Stratified for risk group, the higher percentage of MCR with imatinib resulted in an estimated 10-year survival probability of $66 \%$ for imatinib-treated low-risk patients compared to $37 \%$ for interferon (median survival time: 95 months) (Figure 2); the corresponding figures for intermediate-risk patients were 53 vs 22\% (median survival time: 72 months) (Figure 3). A separate analysis for high-risk patients was not performed, since no relevant increment in survival time was seen for these patients when treated with IFN.

In contrast to IFN-based treatments, the MCR rate of patients treated with imatinib is much higher. However, due to the relatively short observation period, long-term survival with imatinib treatment is not yet known. However, physicians and patients need to know how the higher MCR rate achieved with imatinib will most likely translate into survival time. We combined the survival increments seen in IFN-treated CML patients stratified for prognostic groups and MCR (yes, no), with the higher MCR rates seen in patients treated with imatinib in the randomized IRIS trial. ${ }^{2}$ This is the first data-based estimation of the expectable survival benefits for chronic phase CML patients treated with imatinib.

The expected absolute increment of the 10-year survival rate by $25-31 \%$ is a rather conservative estimate, since we assumed for imatinib at the landmark 21 months identical survival probabilities around $95 \%$ as were observed with IFN. Besides, we used the MCR rate achieved with imatinib until month 18 (as the MCR rate at month 21 was not available) and assumed for the total sample that in imatinib-treated high-risk patients as in those treated with IFN, MCR does not improve survival. MCR rather than complete cytogenetic remission (CCR) rate was applied, since the frequency of CCR (until 21 months: $n=61$; $9.3 \%$ of 658 ) in the IFN sample was too low to be used for such computations. However, for imatinib, a CCR rate of $73.8 \%$ was reported and, in general, an even greater increment in survival time in patients with CCR is expected.

It is still not known whether MCR under imatinib generates the same survival increment as seen with IFN, but quantitative $\mathrm{PCR}$ analyses indicated that the $\mathrm{Ph}+$ clone is even more 
Table 1 New CML score (5) and MCR in 658 patients who were under observation for at least 21 months

\begin{tabular}{|c|c|c|c|}
\hline & No. of patients & Median survival & 10-year survival probability $(\mathrm{Cl})^{\mathrm{a}}$ \\
\hline Low-risk patients/MCR up to month 21 & $88(16 \text { died })^{b}$ & Not reached & $0.74(0.63 ; 0.85)$ \\
\hline Low-risk patients/no MCR up to month 21 & 181 (81 died) & $78 \mathrm{mo}$ & $0.20(0.10 ; 0.31)$ \\
\hline Intermediate-risk patients/MCR up to month 21 & 75 (18 died) & Not reached & $0.60(0.45 ; 0.75)$ \\
\hline Intermediate-risk patients/no MCR up to month 21 & 239 (157 died) & $66 \mathrm{mo}$. & $0.12(0.05 ; 0.19)$ \\
\hline High-risk patients/MCR up to month 21 & 8 (8 died) & $38 \mathrm{mo}$. & $0(0 ; 0)$ \\
\hline High-risk patients/no MCR up to month 21 & 67 (56 died) & $43 \mathrm{mo}$. & $0.11(0.01 ; 0.20)$ \\
\hline
\end{tabular}

Mo. $=$ months

aStandard 95\% confidence intervals.

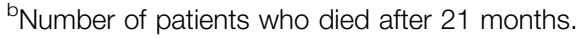

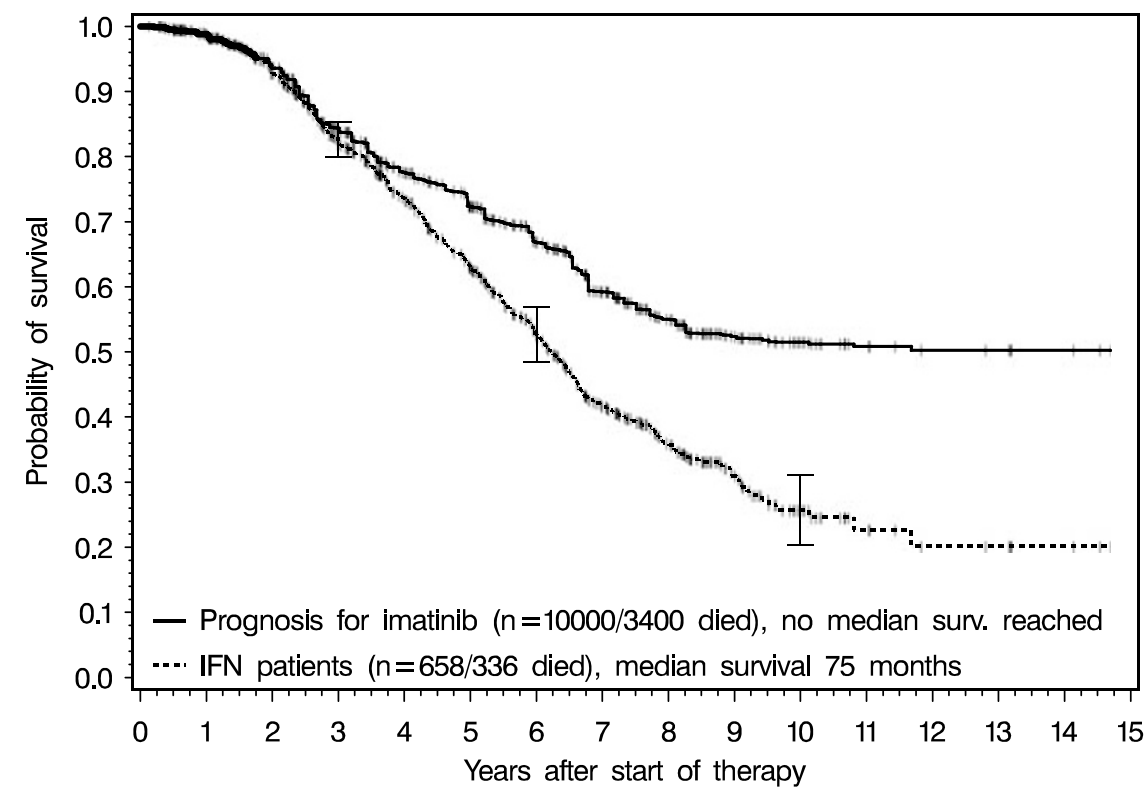

Figure 1 Total patient sample. Prognosticated survival probabilities for imatinib treatment vs survival estimated under IFN treatment.

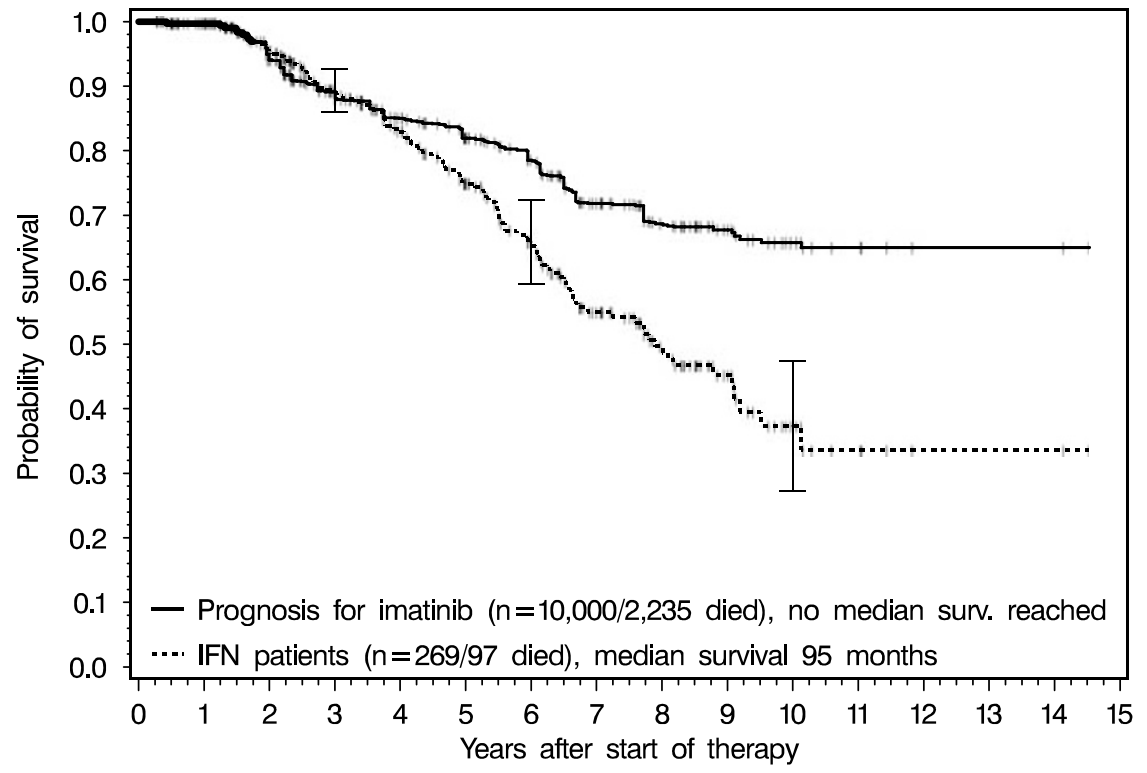

Figure 2 Low-risk patients according to the New CML score. Prognosticated survival probabilities for imatinib treatment vs survival estimated under IFN treatment. 


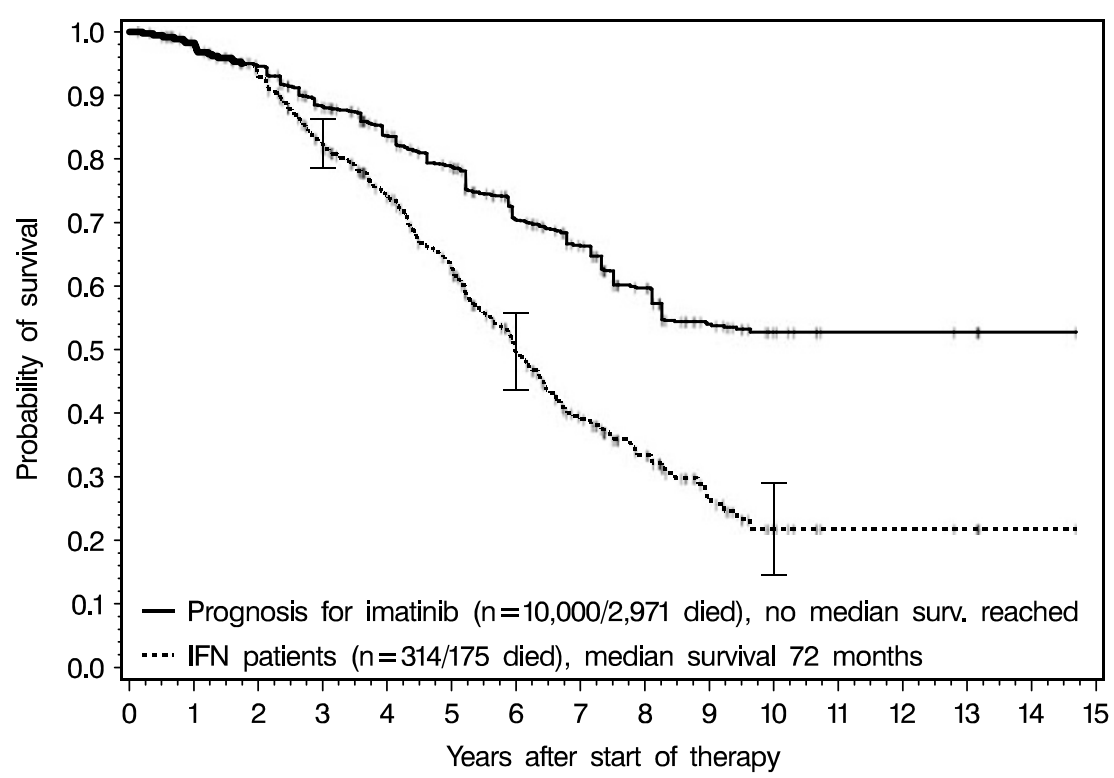

Figure 3 Intermediate-risk patients according to the New CML score. Prognosticated survival probabilities for imatinib treatment vs survival estimated under IFN treatment.

reduced with imatinib than with $\operatorname{IFN}^{6,7}$ and first reports suggested that cytogenetic response is a predictor for survival under imatinib, too. ${ }^{8}$ Emerging evidence that imatinib rarely leads to molecular complete remission and that many patients are still at risk of relapse and other clonal disorders is of concern $^{9,10}$ and emphasizes the need for long-term data of effectiveness.

As imatinib was introduced into the treatment of CML since 2001 only, it will take some years before valid survival time data will be available. For the time being, we consider the expected outcome data presented here as the methodologically best and fair estimates.

\section{Acknowledgements}

This work was supported by an unrestricted grant from Novartis Ltd.

\section{References}

1 Stone RM. Optimizing treatment of chronic myeloid leukemia: a rational approach. Oncologist 2004; 9: 259-270.

2 O'Brien SG, Guilhot F, Larson RA, Gathmann I, Baccarani M, Cervantes F, et al., IRIS Investigators. Imatinib compared with interferon and low-dose cytarabine for newly diagnosed chronic phase chronic myeloid leukemia. N Engl J Med 2003; 348: 994-1004.
3 Kantarjian HM, Smith TL, O'Brien S, Beran M, Pierce S, Talpaz M. Prolonged survival in chronic myelogenous leukaemia after cytogenetic response to interferon- $\alpha$ therapy. Ann Intern Med 1995; 122: 254-261.

4 Bonifazi F, de Vivo A, Rosti G, Guilhot F, Guilhot J, Trabacchi E et al. Chronic myeloid leukemia and interferon-alpha: a study of complete cytogenetic responders. Blood 2001; 98: 3074-3081.

5 Hasford J, Pfirrmann M, Hehlmann R, Allan NC, Baccarani M, Kluin-Nelemans JC et al. A new prognostic score for survival of patients with chronic myeloid leukemia treated with interferon alfa. J Natl Cancer Inst 1998; 90: 850-858.

6 Hughes TP, Kaeda J, Branford S, Rudzki Z, Hochhaus A, Hensley $\mathrm{ML}$ et al. Frequency of major molecular responses to imatinib or interferon alfa plus cytarabine in newly diagnosed patients with chronic myeloid leukemia. N Engl J Med 2003; 349: 1423-1432.

7 Müller MC, Gattermann N, Lahaye T, Deininger MWN, Berndt A, Fruehauf $S$ et al. Dynamics of BCR-ABL mRNA expression in first line therapy of chronic myelogenous leukemia patients with imatinib or interferon $\alpha /$ ara-C. Leukemia 2003; 17: 2392-2400.

8 Kantarjian HM, Cortes JE, O'Brien S, Luthra R, Giles F, Verstovsek $\mathrm{S}$ et al. Long-term survival benefit and improved complete cytogenetic and molecular response rates with imatinib mesylate in Philadelphia chromosome-positive, chronic-phase chronic myeloid leukemia after failure of interferon-(alfa). Blood 2004; 104: 1979-1988.

9 Angstreich GR, Smith BD, Jones RJ. Treatment options for chronic myeloid leukemia: imatinib versus interferon versus allogeneic transplant. Curr Opin Oncol 2004; 16: 95-99.

10 Hochhaus A, La Rosée P. Imatinib therapy in chronic myelogenous leukemia: strategies to avoid and overcome resistance. Leukemia 2004; 18: 1321-1331. 